\title{
A Tentative Analysis of Errors in Language Learning and Use
}

\author{
Wenfen Yang \\ School of Foreign Languages, Qingdao University of Science \&Technology, Qingdao, China \\ Email: wfyoung@163.com
}

\begin{abstract}
Everyone tends to make mistakes in the process of language learning and use, especially for FL learners. This paper makes a tentative effort to describe and diagnose learners' errors in language learning and use in the hope of helping FL learners' have a better understanding of the errors they made.
\end{abstract}

Index Terms - error, language learning, language use, EA, CA

As human beings, we are bound to make mistakes. If to err and to speak are each uniquely human, then to err at speaking, or to commit language errors, must mark the very pinnacle of human uniqueness. Since language error is the subject of this paper, let's provisionally define a language error as an unsuccessful bit of language. Error Analysis is the process of determining the incidence, nature, causes and consequences of unsuccessful language. The novelty of EA, distinguishing it from CA, was that the mother tongue was not supposed to enter the picture. Errors could be fully described in terms of the TL, without the need to refer to the L1 of the learners.

The Error Analyst's object of enquiry is the FL learner's ignorance of the TL. This ignorance can be manifest in two ways. First in silence, then in the way they compensate for their ignorance, i.e. substitutive language. We need to distinguish two sorts of silence: cultural silence and avoidance. Cultural silence caused by EL2 learners from 'silent cultures' such as Finns or Japanese; while avoidance is induced by ignorance. But learners usually prefer to try to express themselves in the TL by alternative means: they 'beg, steal or borrow'. This is what we have said before the substitutive language (called IL). Error Analysts study it in relation to the TL.

\section{THE DESCRIPTION OF ERROR}

\section{A. Error Detection}

In the criminal investigation, a line-up of individuals is assembled and then let an eyewitness to pick out the perpetrator of the crime. Analogically, in EA we assemble a line-up of utterances produced or processed by a learner and ask the 'witness' or knower to pick out the one or ones that look suspicious, that is, those which are potentially erroneous. You may think error detection is simple, but in fact it is just the opposite. It is interesting to find out that to spot error in spoken, informal language is harder than in written, formal texts. In addition, spotting one's own errors is more difficult than spotting other people's errors. Even the native speakers sometimes cannot detect error correctly.

\section{B. Locating Errors}

Error location is in the same way with what the police ask an eyewitness to identify the suspect, such as saying he is the third from the left. However, error location is not always so straightforward; some errors are diffused throughout the sentence or larger unit of text that contains them: they are known as global errors. Burt and Kiparsky (1972) suggest that we should identify errors by reference to the TL. And there is a reasonable suggestion: the learners first need to stop making the error, and then start to produce the TL form. Gatbonton (1983) adopts the gradual diffusion model of language change, which suggests that learning involves two sages: the first is the learning stage and the second involves extirpating all the old and wrong learning so only the good ones are left.

\section{Describing Errors}

There are two reasons for why the standard EA practice of describing the learner's errors should be in terms of the TL: firstly, learner's language and TL are co-dialects of the same language, so they should be describable in terms of the same grammar. Secondly, EA is TL-oriented. According to Corder (1981), the system used for the description of learner's errors must be one having two essential characteristics: 1) the system must be well-developed and highly elaborated, since many errors made by even beginners are remarkably complex. 2) The system should be as simple, self-explanatory and easily learnable as possible. Because of the two characteristics James (1990) points out that Chomsky's Universal Grammar has little usage for describing learner errors.

\section{LEVELS OF ERROR}

In this part, we recognize three levels of language: the levels of substance, text and discourse. If the learner was operating the phonological or the graphological substance systems, that is spelling or pronouncing, we say he or she has 
produced an encoding or decoding error. If he or she was operating the lexico-grammatical systems of the TL to produce or process text, we refer to any errors on this level as composing or understanding errors. If he or she was operating on the discourse level, we label the errors occurring misformulation or misprocessing errors.

\section{A. Misspellings.}

There are four types of misspelling: the first one is punctuation errors, among which the most frequent are overuse of the exclamation (!) by some writers; misordering of closing inverted comas; under or overuse of capitals; over inclusion of a comma between an antecedent and a restrictive relative clause; and misselection of the colon instead of the comma after the salutation in letters. The second one is typographic errors. People who are normally good spellers might be poor typists. Their problem is in automatizing the required temporal and spatial mechanisms that underlie skilled fingering on the typewriter, or keystrokes on the word-processor. In fact, we are dealing here with mistakes rather than errors, and this probably explains why psychologists interested in skill learning have paid more attention to typing errors than applied linguists have. The third on is dyslexic errors. Some of the errors dyslexics make are MSs: e.g. <parc> for <park> is a misselection from two letters that can represent the same sound 〔k〕 in English. Dyslexics also produce errors that are not MSs, e.g. $\langle\mathrm{deb}\rangle$ for $\langle\mathrm{bed}\rangle$ involving the reversal of the letter $\langle\mathrm{b}\rangle$ into $\langle\mathrm{d}\rangle$ or strephosymbolia. The fourth one is confusibles. Let's look at the amusing example given by Carney (1994): 'to marry a devoiced woman' and observes that these are lexical errors involving confusion between similar sounding morphemes and words. Perhaps literacy raises people's awareness of differences to the point where they can avoid such confusions in their writing at least.

\section{B. Lexical Errors.}

According to Chomsky (1980) lexis is sharply different from grammar. Grammar is said to be organized in closed systems, to be systematic and regular. Lexis is, by contrast, said to consist of open systems, to be irregular and unsystematic. Recently, lexis has begun to take a central role in language study. There are a number of reasons for this. First, the boundaries between lexis and grammar are now seen to be less clear-cut than was assumed. Morphological aspects of words, which used to be treated as part of grammar, can just as well be viewed as part of the word: e.g. words of different form classes can be derived from the same root: bright $\rightarrow$ brightness $\rightarrow$ brightly. Secondly, learners themselves believe that vocabulary is very important in language learning. Though this view may not be correct, it is likely to influence learning. Thirdly, for some learner groups, lexical errors are the most frequent category of error. Fourthly, native speakers consider the lexical errors in learners' IL to be more disruptive and irritating than other types. Finally, vocabulary carries a particularly heavy functional load, especially in early IL. There is little grammar in such IL, and the message often has to be inferred, mainly from the lexical terms assembled for its representation.

We classify lexical errors from two perspectives: formal errors and semantic errors. Formal errors of lexis include formal misselection, misformations and distortions. Semantic errors in lexis refer to confusion of sense relations and collocational errors.

\section{Pragmatic Errors.}

Pragmatic errors involve putting linguistic knowledge into practice, so we may call them pragmalinguistic deviations. They arise when ever speakers misencode a message, not to the detriment of its meaning but to the detriment of its pragmatic force, that is, what speech act it is intended to perform or what rhetorical force it should carry. On the other hand, there are discoursal or interactional gaffes that arise not out of linguistic incompetence but out of sociocultural incompetence. Thomas (1983) calls it sociopragmatic failure. Sociopragmatic failures result from culture-clashes, from cultural differences of view concerning what is appropriate social behavior in certain settings. There are a number of sources of such infelicities, such as taboos, size of the imposition, values and power and social distance.

\section{DIAGNOSING ERRORS}

James (1990) once commented on the desirability of distinguishing between error description and error diagnosis. There is widespread acceptance of this principle: Dulay, Burt and Krashen (1982) clearly state that 'the accurate description of errors is a separate activity from the task of inferring the sources of those errors'. We might ask what motivates this insistence on keeping description and diagnosis separate. To illustrate it more clearly, let's look at how doctors diagnose diseases: sick patients visiting the doctor show visible signs of their illnesses, which the doctor describes in order to reach a diagnosis. In addition, the patients are usually asked to describe their sensation to the doctor, who interprets this account in terms of a diagnosis. The same is true for EA. The diagnosis question is therefore one that transcends description and invokes explanation, tracing errors to their causes, sometimes called error etiology.

The ultimate cause of error is ignorance of the TL item aimed at. It is worth noting that any formal deviance can have either declarative or procedural causes. When the required TL item is unknown and the learner borrows an L1substitute, the consequence is an L1 transfer error, but when the learner knows the TL item but fails to access it, and instead accesses an L1 substitute, we have a case of an L1 interference mistake.

There is general agreement over the main diagnosis-based categories of error. There are four major categories: interlingual, intralingual, communication-strategy and induced. 
The clearest proof of L1 interference is where L1 nonstandard dialect features get transferred to L2. let's look at an example made by L1 Portuguese learners of EL2: the fronted preposition in With who did he come? Ninety-nine per cent of the time the learners might prefer: Who did he come with? Now when they come to learn French or German, they will have a problem, since in these languages the preposition must be fronted. Failure to do so results in errors like Wem ist er gekommen*mit?

Intralingual errors are based on learning strategy. The author gives us a list of errors. We will discuss some of them: 1) false analogy: the learner wrongly assumes that the new item B behaves like A, e.g. boy $\rightarrow$ boys, then child $\rightarrow$ * childs. 2) Misanalysis: the learners have formed a hunch or hypothesis concerning an L2 item, which they are now putting into practice. The hypothesis is not based on L1 knowledge at all, e.g. 'Tinker, Tailor', as every story *who tells about spies... The learner has hypothesized that since the book's title refers to humans, therefore the $(+$ human relative pronoun should be selected. 3) Hypercorrection: this results from the learners over-monitoring their L2 output, and attempting to be consistent, so it is akin to system simplification: II est descendu et *est (a) attendu. The learner seems to have learnt that the marked auxiliary etre is used with descendre but wrongly assumes that it should also be used with attendre.

Communication strategy-based errors include holistic strategies and analytic strategies. The term 'holistic' refers to the learners' assumption that if you can say X in the L2, then you must be able to say Y. The most general term for this is approximation. It takes on a number of forms, the first of which is to use a near synonym, e.g.* credibility for the intended truth. Alternatively, one can use a superordinate term: *fruits for blackberries. A third option is to use an antonym or opposite, and fourth option is to coin a word. Analytic strategies express the concept indirectly, by allusion rather than by direct reference: this is circumlocution. The learners identify one or more criterial attributes of the referent and mention these in an attempt to refer to the entity in question.

Induced errors was first used by Stenson (1983) to refer to learner errors 'that result more from the classroom situation than from either the students' incomplete competence in English grammar or first language interference'. Some of what Stenson (1983) calls induced errors could be explained in terms of the diagnostic categories we have outlined above. Similarly, there are those errors induced by imprecise teacher explanations: for example, the pedagogical 'explanation' which distinguishes the modals should and must on the grounds that the former is 'stronger' than the second. The error precipitated was we *should have worked in order to buy clothes, but we *must have worked in order to eat. The learners are attempting to convey the idea that it is less important to spend money on clothes than on food. In fact the learners have hypothesized a false concept not on the basis of their own analysis of input but on that of their teacher's mediation of the lexical opposition.

\section{CONCLUSION}

Error analysts claim that learners' errors, to a large degree, are not caused by the influence of their L1; instead, their errors reflect some common learning strategies. EA tries to find out the regular things in the process of foreign language learning through the study of learners' errors. In this way it has a great contribution for FL teaching: teachers should be sensitive to their students' errors and summarize what kind of error students are most likely to make at certain period, and then modify their teaching materials in order to adapt to the students' need.

\section{REFERENCES}

[1] Burt, M. and Kiparsky, C. (1972). The Gooficon: A Repair Manual for English. Newbury House, Rowley, MA.

[2] Carney, E. (1994). A Survey of English Spelling. Routledge, London.

[3] Chomsky, N. (1980). Rules and Representations. Blackwell, Oxford.

[4] Corder, S.P. (1981). Error Analysis and Interlanguage. Oxford University Press, Oxford.

[5] Dulay, H., Burt, M. and Krashen, S.D. (1982). Language Two. Newbury House, Rowley, MA.

[6] Gatbonton, E. (1983). Patterned phonetic variability in second language speech: a gradual diffusion model, in B.W. Robinett and J. Schachter (eds), Second Language Learning: Contrastive Analysis, Error Analysis and Related Aspects. University of Michigan Press, Ann Arbor, MI. pp. 240-55.

[7] James, Carl. (1990). Learner language, Language Teaching Vol. 23 No. 4: 205-13.

[8] James, Carl and Garrett, P. (eds) (1991). Language Awareness in the Classroom. Longman, London.

[9] Stenson, N. (1983). Induced errors, in B.W. Robinett and J. Schachter (eds), Second Language Learning: Contrastive Analysis, Error Analysis and Related Aspects. University of Michigan Press, Ann Arbor, MI. pp. 256-71.

[10] Thomas, J. (1983). Cross-cultural pragmatic failure, Applied Linguistics Vol. 4 No. 2: 91-112.

Wenfen Yang was born in Shandong, China in 1965. She received her M.A. degree in linguistics from Shandong Teachers' University, China in 1993.

She is currently an associate professor in the School of Foreign Languages, Qingdao University of Science \& Technology, Qingdao, China. Her research interests include linguistics and translation. 